\title{
Modeling the electric field induced in a high resolution realistic head model during transcranial current stimulation
}

\author{
R. Salvador, A. Mekonnen, G. Ruffini, P. C. Miranda
}

\begin{abstract}
Much of our knowledge about the electric field distribution in transcranial current stimulation (tCS) still relies on results obtained from layered spherical head models. In this work we created a high resolution finite element model of a human head by segmentation of MRI images, and paid particular attention to the representation of the cortical sheet. This model was then used to calculate the electric field induced by two electrodes: an anode placed above the left motor cortex, and a cathode placed over the right eyebrow. The results showed that the maxima of the current density appear located on localized hotspots in the bottom of sulci and not on the cortical surface as would be expected from spherical models. This also applies to the components of the current density normal and tangential to the cortical surface. These results show that such highly detailed head models are needed to correctly predict the effects of tCS on cortical neurons.
\end{abstract}

\section{INTRODUCTION}

$\mathrm{T}_{\mathrm{n}}^{\mathrm{p}}$ RANSCRANIAL stimulation with weak slowly modulated electrical currents (tCS) is a promising technique that has been shown to modulate cortical excitability in humans [1]. Modulation of the cortical neurons by tCS depends on the induced electric field in the brain. Much of our knowledge about the spatial distribution of the electric field during transcranial electrical stimulation still comes from early calculations performed using simplified head models. One of the best known and successful model of this kind is the layered spherical head model with point-like electrodes [2], [3]. Similar models that include more layers and more realistic electrodes are still being used in a number of studies [4], [5]. With the advent of powerful computers, more realistic head models have been created from segmentation of high resolution anatomical images obtained from MRI and CT scans [6], [7].

Here we present a finite element calculation of the electric field induced during tCS in a high resolution realistic head model obtained from segmentation of the Colin27 template. Special care was taken to accurately represent the cortical

The project HIVE acknowledges the financial support of the Future and Emerging Technologies (FET) programme within the Seventh Framework Programme for Research of the European Commission, under FET-Open grant number: 222079.

R. Salvador, A. Mekonnen and P. C. Miranda are with the Institute of Biophysics and Biomedical Engineering (IBEB), Faculty of Sciences, Lisbon 1749-016, Portugal (phone: +351217500177; fax: +351217500030 ; e-mail: rnsalvador@fc.ul.pt). G. Ruffini is with the Starlab, Barcelona, Spain. sulci since these can significantly affect the electric field distribution in the brain.

\section{METHODS}

\section{A. Creation of the head model}

The realistic head model for the calculation of the electric field was build in four steps: selection of suitable MR images, segmentation, generation of meshes representing the relevant surfaces and generation of volume meshes, and use of the volume meshes in a finite element program to calculate the electric field. The images used for the present model were T1 and PD phantom images based on the Colin27 template, (obtained from BrainWeb, http:/www.bic.mni.mcgill.ca/brainweb/). Segmentation was performed using the software BrainSuite (http://www.loni.ucla.edu/Software/BrainSuite): white matter (WM), gray matter (GM) and cerebrospinal fluid (CSF) were segmented from the T1 images whereas the skull and scalp were obtained from the PD images. This information was then imported into MIMICS (http://www.materialise.com/) where volume meshes suitable for finite element calculations were generated (see Fig. 1). The meshes were then exported into a finite element program used to calculate the electric field: Comsol (v. 3.5a www.comsol.com). The resultant mesh comprises more than $1.9 \times 10^{6}$ tetrahedral second order Lagrange elements.

\section{B. Electrode design and placement}

Two $25 \mathrm{~cm}^{2}$ electrodes were also represented in the model (see Fig. 1a). The electrodes were designed with a cylindrical shape (radius of $28.2 \mathrm{~mm}$ ) and a height of approximately $1.5 \mathrm{~cm}$. A perfect electrical contact was assumed between the electrodes and the scalp.

The anode was placed over the hand area in the left motor cortex, whereas the cathode was placed over the right eyebrow. Identification of the "hand-knob" [8] in the motor cortex was done by visual inspection of the anatomical images.

The upper surface of each electrode was set to uniform electrical potential and the potential difference between them was adjusted so that the current injected through the anode was $1.0 \mathrm{~mA}$. All the remaining outer boundaries of the model were considered to be insulating $(\vec{J} \cdot \vec{n}=0)$, and continuity of the normal component of the current density was imposed on all the inner boundaries. 


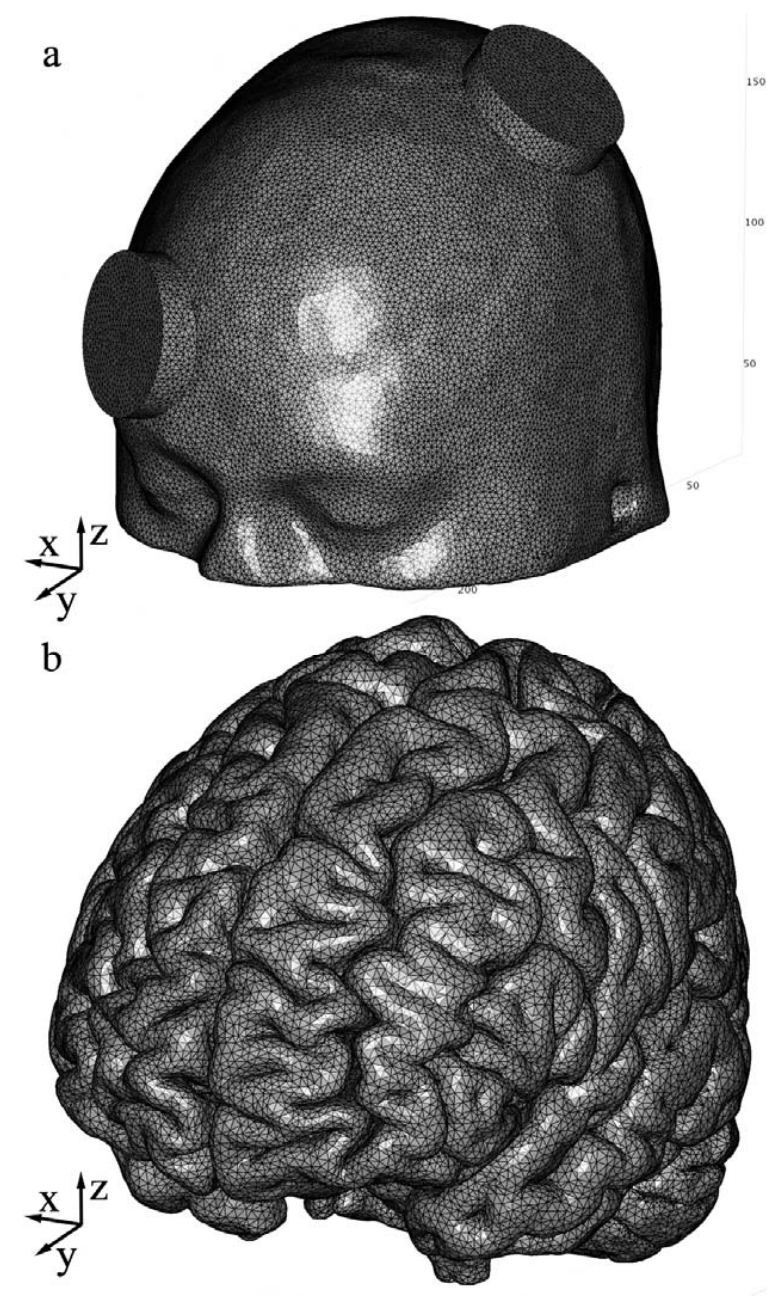

Fig. 1. Finite element mesh of the head model used in this work. (a): scalp and electrodes; (b): interface between GM and CSF. In the model, the $x$ axis points in the left to right direction, the $y$ axis in the posterior-anterior direction and the $z$ axis in the inferior-superior direction.

\section{Electric field calculation}

The electric field induced in the brain was obtained by solving Laplace's equation $(\nabla \cdot(\sigma \nabla \phi)=0$, where $\phi$ is the electrostatic potential and $\sigma$ is the electric conductivity) and taking the gradient of the scalar potential. This procedure assumes that the quasi-statics approximation holds [9]. In this approximation the tissues are considered to be purely resistive with no capacitive components. This approximation is valid for the low stimulation frequencies used in tCS.

The different tissues in the head model were modeled as having electrical conductivities with values close to the average ones reported in the literature for the DC / low frequency range [10]-[12]: $0.32 \mathrm{~S} / \mathrm{m}, 0.008 \mathrm{~S} / \mathrm{m}, 1.79 \mathrm{~S} / \mathrm{m}$, $0.32 \mathrm{~S} / \mathrm{m}$ and $0.15 \mathrm{~S} / \mathrm{m}$ for the scalp, skull, CSF, GM and $\mathrm{WM}$, respectively. The electrodes were modeled as having a conductivity value arbitrarily taken to be equal to that of the scalp [4]. All media were modeled as having isotropic conductivities and, as such, the current density could be found simply by multiplying the scalar electrical conductivity by the electric field.
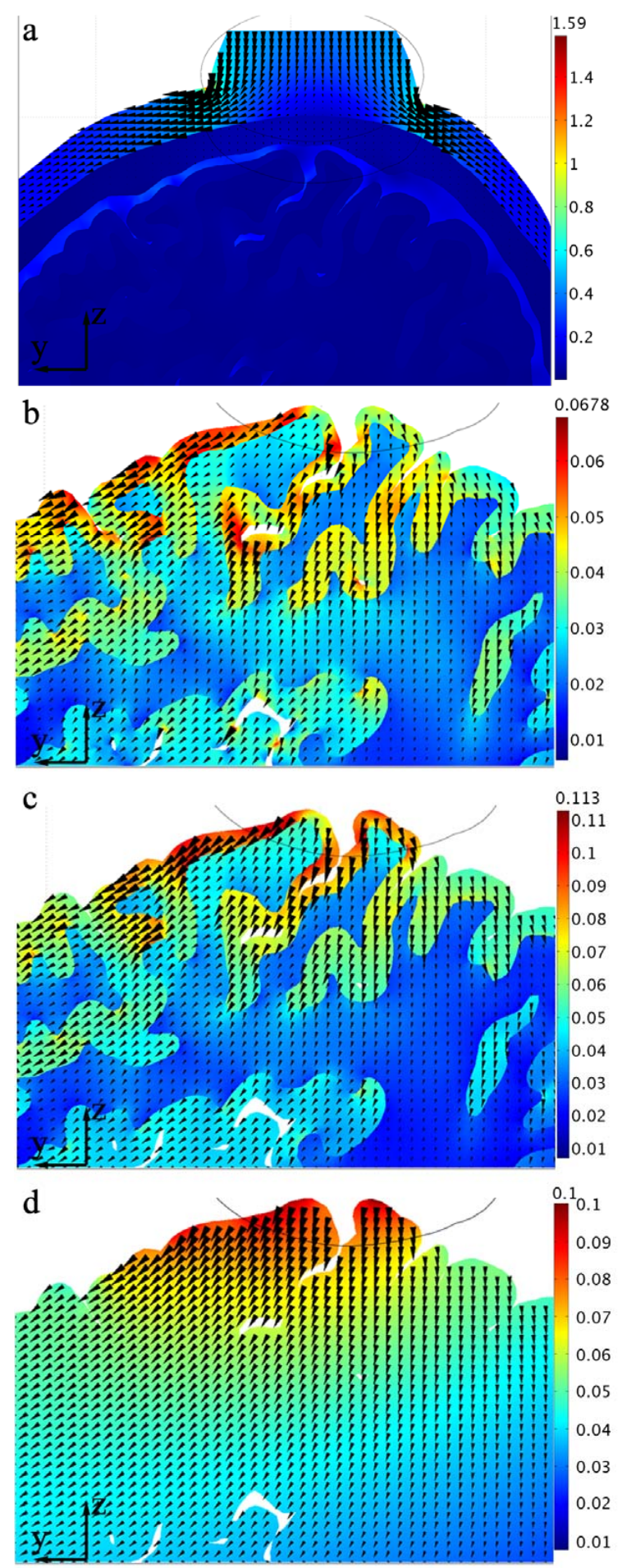

Fig. 2. Spatial distribution of the magnitude of the current density in a sagittal slice located under the anode $(x=50 \mathrm{~mm})$. The image shown in (a) includes all the tissues in the model as well as the anode. The image in (b) shows only the current density distribution in the GM and WM. The image shown in (c) is the same as the one shown in (b) but now for a model in which the conductivity of the CSF is set to the same value as that of the GM. Finally, the image shown in (d) is the same as the one shown in (b)/(c) but now for a model with the conductivity of the CSF and WM set to the same value as that of the GM. The color bars represent the norm of the current density in $\mathrm{A} / \mathrm{m}^{2}$. 


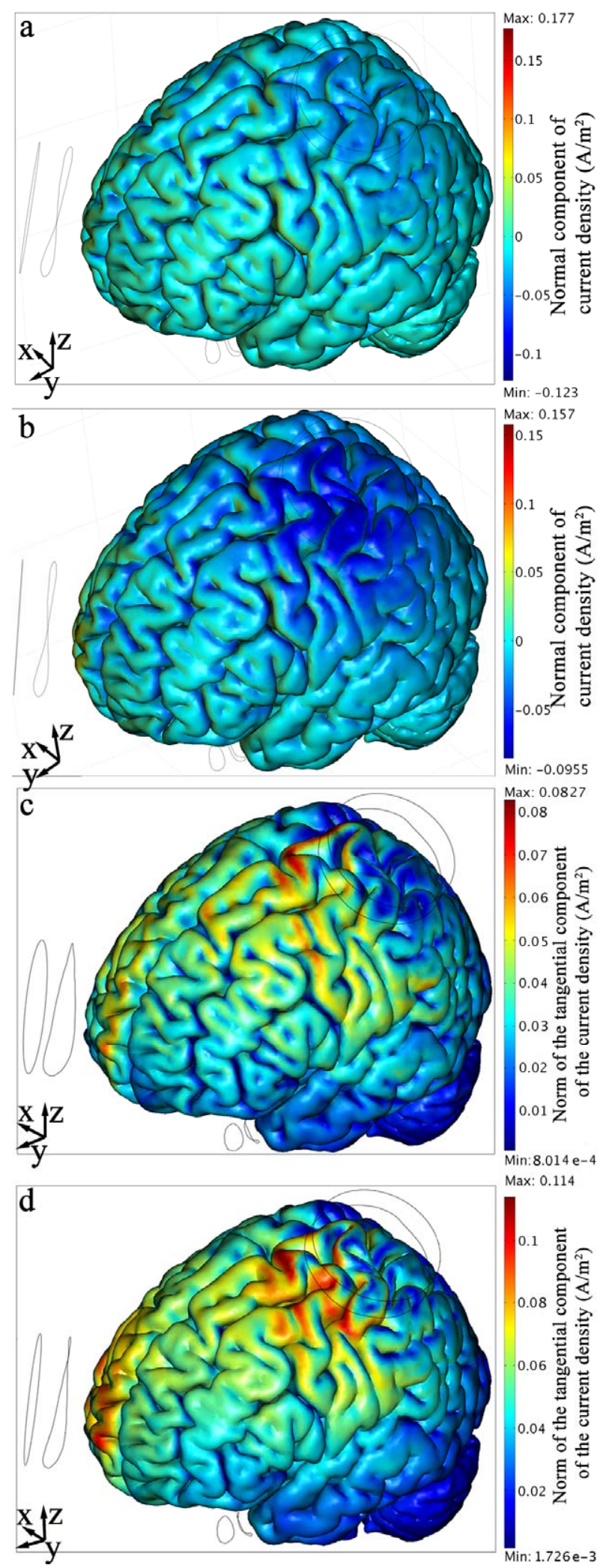

Fig. 3. Distribution of the normal $(\mathrm{a} / \mathrm{b})$ and tangential $(\mathrm{c} / \mathrm{d})$ components of the current density on the GM - CSF interface. (a) and (c): Model with all the tissues with appropriate conductivity values. (b) and (d): Model with the CSF's conductivity equal to that of the GM. Note that, as the component of the current density tangential to the GM - CSF interface is discontinuous, we chose to represent the value on the GM side of the interface.
All calculations were performed in Comsol, using its Conductive Media DC package. Given the large number of degrees of freedom in the model (more than $5 \times 10^{6}$ ), an iterative linear system solver (GMRES) was chosen. This iterative solver required that a preconditioner be used (Incomplete LU with a drop tolerance of 0.001). The calculation took about 3 hours to complete in a workstation with two quad-core Xeon W5580 processors at $3.2 \mathrm{GHz}$ and 48 GB of RAM memory.

\section{RESULTS}

\section{A. Spatial distribution of the current density}

The magnitude of the current density is greatest on the scalp under the electrodes but it decreases rapidly with depth. For the sagittal plane depicted in Fig. 2, the current density norm on the scalp reaches a maximum of $1.6 \mathrm{~A} / \mathrm{m}^{2}$ (Fig. 2a), but this value decreases to $0.33 \mathrm{~A} / \mathrm{m}^{2}$ on the CSF and only $0.068 \mathrm{~A} / \mathrm{m}^{2}$ on the GM (Fig. 2b). The shunting effect that the scalp exerts on the current is also clearly visible in Fig. 2a. Another interesting fact is that the current density is highest on the scalp under the rim of the electrode and is not uniformly distributed under the electrode (see Fig. 2a). This explains why the maximum value of the current's density norm in the scalp is much higher than that predicted by dividing the total injected current by the area of the electrode $\left(0.40 \mathrm{~A} / \mathrm{m}^{2}\right)$. This shows that the ratio injected current / electrode area does not predict accurately the current density in the scalp [13].

Contrary to what was expected, the maximum of the current density induced on the GM - CSF interface does not occur on the gyri close to the electrode but instead at localized hotspots, some localized on the bottom of sulci (see Fig. 2b). This seems to be a result of the shunting effect of the CSF because when the conductivity of the CSF is set to a value equal to that of the GM no such hotspots appear on the bottom of sulci (see Fig. 2 c), although some still appear at the wall of the sulci. Setting the conductivity of all tissues represented in the model, except the skull, to the same value as that of the GM restricts the position of the maxima to the top of the gyri, closer to the electrodes (see Fig. 2 d), as predicted by the spherical models.

These observations apply also to the component of the current density perpendicular to the cortical surface, as is illustrated in Fig. 3a. Again, setting the conductivity of the CSF to that of the GM shifts the maxima to the gyri close to the electrodes (compare Fig. 3a and b). Regarding the component of the current density tangential to the cortical surface, the presence of the CSF also seems to shift the maxima to more deeply located regions in the walls of the sulci, as can be seen by comparing Fig. $3 \mathrm{c}$ and d. There is, however, one maximum that appears at the cortical surface in the model with the appropriate conductivity value for the CSF. This maximum is located under the rim of the anode, at a gyrus located close to the central fissure (see Fig. 3c). It 
should be noted that although this analysis focused essentially on the distribution of the current density under the anode, it also applies to the region under the cathode.

Changes in the electrical conductivity of the skull affect mainly the magnitude of the current density values but have little effect on its spatial distribution. Reducing the conductivity of the skull to half of its original value (this results in a ratio between the conductivity of the scalp and that of the skull of 80 , a value used in some early modeling studies, [3]) reduces the magnitude of the current density in the cortical surface to $63 \%$ of its value with the original conductivity value. The location of the maxima remains at the bottom of the sulci, however.

\section{DISCUSSION}

The analysis of the current density distribution in this high resolution realistic head model yielded some results that were unexpected and very different from those obtained on simpler models. One of these results is that the maxima of the current density norm do not appear on the gyri under the electrodes but in localized hotspots at the bottom of the sulci. This effect disappears when the conductivity of the CSF is set to a value equal to that of the GM and has not been reported in studies involving spherical head models [4], [5]. Therefore, this effect results from a combination of both the high conductivity of the CSF and the highly convoluted surface of the cortex.

Even when the conductivity of the CSF is set to the same value as that of the GM, the distribution of the current density is not the same as that predicted by spherical head models, with maxima still appearing at the walls of the sulci. This effect seems to be a result of the difference in the conductivities of the GM and the WM because when that heterogeneity is removed the distribution of the current density becomes similar to that predicted by the simpler models, with the maxima appearing only at the gyri closer to the electrodes. The fact that the WM is highly anisotropic [14] is likely to increase even more the complexity of the spatial variation of the electric field.

Similar effects were reported in another realistic head model [6]. In this study, the authors noticed that the maximum of the current density / electric field appeared at the walls of some sulci, a phenomenon they called "electric field clustering". The sulci do not seem to be as well represented in that model as they are in the one used in the present work. As a result, the maxima of the current density in our model appear to be located not only at the walls of sulci, but also deeper in the bottom of sulci.

We have also shown that this "clustering" affects the components of the current density normal and tangential to the cortical surface. These are thought to be the relevant components in modeling the effects of tCS on cortical neurons, given that neurons tend to align mainly in directions either normal or tangential to the cortical surface [15]. The present study demonstrates clearly the necessity of employing highly detailed realistic head models to correctly determine which cortical sites are more likely to be influenced by the electric field induced in tCS. Our model can also be used to investigate similar effects in TMS.

In this work, the electric field and the current density in a given tissue have the same distribution because tissues were modeled as isotropic. In the future, we intend to include tissue anisotropy in our models.

This preliminary work was carried out under the EC funded HIVE project to model the effects of multi-site noninvasive electrical brain stimulation.

\section{REFERENCES}

[1] M. A. Nitsche, and W. Paulus, "Excitability changes induced in the human motor cortex by weak transcranial direct current stimulation," Journal of Physiology-London, vol. 527, no. 3, pp. 633-639, Sep 15, 2000.

[2] S. Rush, and D. A. Driscoll, "Current distribution in the brain from surface electrodes," Anesth Analg, vol. 47, no. 6, pp. 717-23, NovDec, 1968.

[3] S. Rush, and D. A. Driscoll, "Eeg Electrode Sensitivity - an Application of Reciprocity," Ieee Transactions on Biomedical Engineering, vol. Bm16, no. 1, pp. 15-\&, 1969.

[4] P. C. Miranda, M. Lomarev, and M. Hallett, "Modeling the current distribution during transcranial direct current stimulation," Clin Neurophysiol, vol. 117, no. 7, pp. 1623-9, Jul, 2006.

[5] A. Datta, M. Elwassif, F. Battaglia et al., "Transcranial current stimulation focality using disc and ring electrode configurations: FEM analysis," Journal of Neural Engineering, vol. 5, no. 2, pp. 163-174, Jun, 2008.

[6] A. Datta, V. Bansal, J. Diaz et al., "Gyri-precise head model of transcranial direct current stimulation: Improved spatial focality using a ring electrode versus conventional rectangular pad," Brain Stimulation, vol. 2, no. 4, pp. 201-207, Oct, 2009.

[7] M. Chen, and D. J. Mogul, "A structurally detailed finite element human head model for simulation of transcranial magnetic stimulation," Journal of Neuroscience Methods, vol. 179, no. 1, pp. 111-120, Apr 30, 2009.

[8] T. A. Yousry, U. D. Schmid, H. Alkadhi et al., "Localization of the motor hand area to a knob on the precentral gyrus - A new landmark," Brain, vol. 120, pp. 141-157, Jan, 1997.

[9] R. Plonsey and D. B. Heppner, "Considerations of Quasi-Stationarity in Electrophysiological Systems," Bulletin of Mathematical Biophysics, vol. 29, pp. 657-\&, 1967.

[10] P. W. Nicholson, "Specific impedance of cerebral white matter," Exp Neurol, vol. 13, no. 4, pp. 386-401, 1965.

[11] S. B. Baumann, D. R. Wozny, S. K. Kelly et al., "The electrical conductivity of human cerebrospinal fluid at body temperature," Ieee Transactions on Biomedical Engineering, vol. 44, no. 3, pp. 220-223, Mar, 1997.

[12] S. Goncalves, J. C. de Munck, J. P. A. Verbunt et al., "In vivo measurement of the brain and skull resistivities using an EIT-based method and the combined analysis of SEF/SEP data," Ieee Transactions on Biomedical Engineering, vol. 50, no. 9, pp. 11241128, Sep, 2003.

[13] P. C. Miranda, P. Faria, and M. Hallett, "What does the ratio of injected current to electrode area tell us about current density in the brain during tDCS?," Clin Neurophysiol, vol. 120, no. 6, pp. 1183-87, May 5, 2009.

[14] C. H. Wolters, A. Anwander, X. Tricoche et al., "Influence of tissue conductivity anisotropy on EEG/MEG field and return current computation in a realistic head model: A simulation and visualization study using high-resolution finite element modeling," Neuroimage, vol. 30, no. 3, pp. 813-826, Apr 15, 2006.

[15] P. T. Fox, S. Narayana, N. Tandon et al., "Column-based model of electric field excitation of cerebral cortex," Hum Brain Mapp, vol. 22, no. 1, pp. 1-14, May, 2004. 